\title{
Globotriaosylsphingosine (lyso-Gb3) might not be a reliable marker for monitoring the long-term therapeutic outcomes of enzyme replacement therapy for late-onset Fabry patients with the Chinese hotspot mutation (IVS4+919G>A)
}

Hao-Chuan Liu' ${ }^{1,2+}$, Hsiang-Yu Lin ${ }^{3,4,5,6+}$, Chia-Feng Yang ${ }^{1}$, Hsuan-Chieh Liao ${ }^{3,7}$, Ting-Rong Hsu ${ }^{1,3}$, Chiao-Wei Lo ${ }^{8}$, Fu-Pang Chang ${ }^{9}$, Chun-Kai Huang ${ }^{1}$, Yung-Hsiu Lu ${ }^{1,2,3}$, Shuan-Pei Lin ${ }^{4,5,6}$, Wen-Chung Yu 2,10,1 $^{*}$

and Dau-Ming Niu ${ }^{1,2,3^{*}}$

\begin{abstract}
Background: In Taiwan, DNA-based newborn screening showed a surprisingly high incidence (1/875 in males and 1/399 in females) of a cardiac Fabry mutation (IVS4 + 919G > A). However, the natural course, long-term treatment outcomes and suitable biomarkers for monitoring the therapeutic outcomes of these patients are largely unknown.

Methods: Fabry disease (FD) patients who had received enzyme replacement therapy (ERT) for more than 1 year were enrolled in this study from December 2008 to April 2013. Periodic echocardiography and serum globotriaosylsphingosine (lyso-Gb3) analysis were carried out. Before and after ERT, left ventricular mass index (LVMI) and serum lyso-Gb3 level were compared and the correlation between the change of LVMI and the change of serum lyso-Gb3 were also analyzed.
\end{abstract}

Results: Thirty-six patients, in four patient groups, were enrolled: (1) 16 males with IVS4 + 919G > A mutation; (2) 7 females with IVS4 + 919G > A mutation; (3) 2 males with classical mutations; and (4) 11 females with classical mutations. The follow-up period was 13-46 months. There were significant LVMI reductions after ERT in all four groups after excluding confounding factors. However, interestingly, serum lyso-Gb3 decreased significantly in the early period after ERT in all groups, but increased gradually after an average of 11.1 months after ERT in late-onset male and female Fabry groups, even when their LVMI still decreased or remained stable. Furthermore, there was no correlation between the change of serum lyso-Gb3 and the change of LVMI in both classical and IVS4 + 919G > A FD patients.

Conclusion: Although lyso-Gb3 has a high diagnostic sensitivity in late-onset Fabry patients and has a good response to ERT during the early stages, it might not be a reliable marker for monitoring the long-term therapeutic outcomes of ERT for late-onset Fabry patients with the Chinese hotspot mutation (IVS4 + 919G > A).

Keywords: Biomarker, Fabry disease, Globotriaosylsphingosine, IVS4 + 919G > A mutation, Outcome

\footnotetext{
*Correspondence: wcyu@vghtpe.gov.tw; dmniu1111@yahoo.com.tw

${ }^{\dagger}$ Equal contributors

${ }^{2}$ Taiwan Clinical Trial Consortium in Fabry Disease, Taipei, Taiwan

'Department of Pediatrics, Taipei Veterans General Hospital, No. 201, Section

2, Shih-Pai Road, Taipei 112, Taiwan

Full list of author information is available at the end of the article
}

\section{Biomed Central}

(c) 2014 Liu et al.; licensee BioMed Central Ltd. This is an Open Access article distributed under the terms of the Creative Commons Attribution License (http://creativecommons.org/licenses/by/4.0), which permits unrestricted use, distribution, and reproduction in any medium, provided the original work is properly credited. The Creative Commons Public Domain Dedication waiver (http://creativecommons.org/publicdomain/zero/1.0/) applies to the data made available in this article, unless otherwise stated. 


\section{Introduction}

Fabry disease (FD) is an X-linked lysosomal storage disorder that results from deficient $\alpha$-galactosidase A ( $\alpha-G a l$ A) activity. This leads to progressive accumulation of globotriaosylceramide (Gb3) and related glycosphingolipids in lysosomes of the heart, kidneys, skin and brain. The clinical manifestations in classical FD patients are angiokeratomas, corneal opacities, hypohidrosis, cardiomegaly, renal impairment, acroparesthesias, gastrointestinal abnormalities and cerebrovascular events [1]. The incidence of FD has been reported as 1 in 40,000-117,000 live births in general population [2,3]. During the past decade, late-onset phenotypes of FD, primarily involving the heart, kidneys or cerebrovascular system, have been reported. Patients with the cardiac variant often lack the classical symptoms of FD and present with left ventricular hypertrophy (LVH), arrhythmias or hypertrophic cardiomyopathy in the fifth to eighth decades of life [4-8].

Our team first discovered the surprisingly high incidence of a cardiac GLA mutation, IVS4 + 919G > A, in Taiwan via newborn screening and further identified this mutation in idiopathic hypertrophic cardiomyopathy patients $[9,10]$. Another DNA-based newborn screening program for this mutation in Taiwan revealed a higher incidence (1/875 in males and 1/399 in females) [11].
Patients who carried the IVS4 + 919G > A mutation and were older than 40 years had a higher prevalence of hypertrophic cardiomyopathy (72\% of males and 35\% of females) [12]. Endocardial biopsy of these patients with hypertrophic cardiomyopathy showed typical FD pathological change and significant Gb3 accumulation in the cardiomyocytes (Figure 1). However, the natural course, long-term treatment outcomes and suitable biomarkers for monitoring the therapeutic outcomes of these patients are largely unknown.

Our previous studies revealed that globotriaosylsphingosine (lyso-Gb3) has high diagnostic sensitivity and correlates with left ventricular mass (LVM), taking into account gender and age in our late-onset Fabry patients [13]. However, its use as a biomarker to monitor the therapeutic outcome of enzyme replacement therapy (ERT) has not been carefully evaluated in our late-onset Fabry patients. Several studies have indicated that serum lyso-Gb3 could be a biomarker for assessing ERT for FD. However, those studies were limited to classical Fabry patients and a short-term (<12 months) follow-up [14-16]. In our study, we evaluated the long-term response of lyso-Gb3 and left ventricular mass index (LVMI) to ERT in Fabry patients with classical or IVS4 + 919G > A mutations.
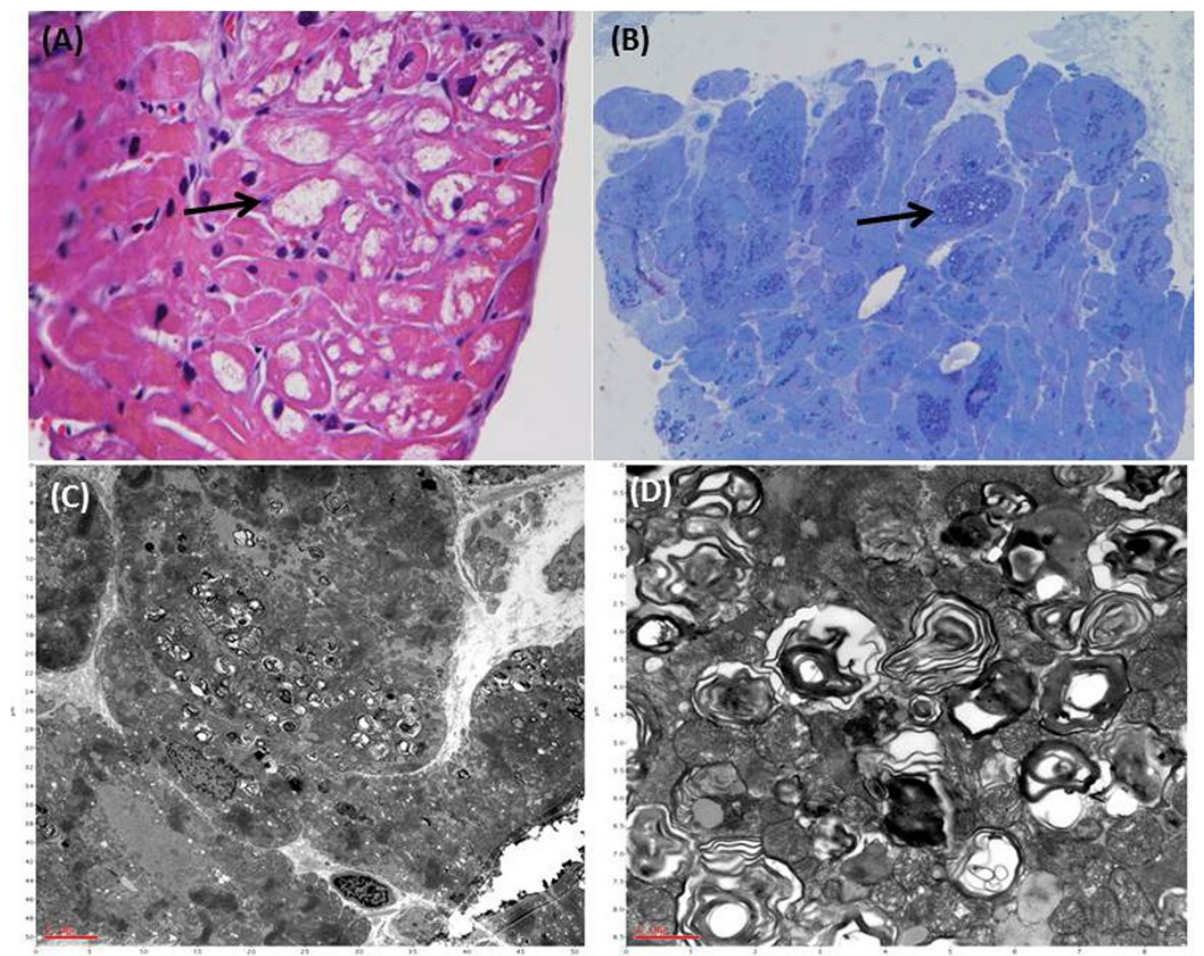

Figure 1 Histological and electron microscopic results of cardiomyocytes of case 20, an IVS4 + 919G > A mutation male, obtained by endocardial muscle biopsy (A) Hematoxylin and eosin (H\&E) staining showed a high degree of vacuolization (arrows) in cardiomyocytes due to lysosomal globotriaosylceramide (Gb3) accumulation. (B) Toluidine blue stain revealed cytoplasmic granular inclusions (arrow) because of Gb3 accumulations. (C) (D) Electron microscopy revealed the characteristic Gb3 concentric lamellar myelin bodies in cardiomyocytes. 


\section{Materials and methods Study population}

This is a retrospective clinical study. From December 2008 to April 2013, all patients at our clinic with significant clinical manifestations of FD, and diagnosis confirmed by plasma $\alpha$-Gal A enzyme assay and GLA gene mutation analysis were eligible for enrollment. Those who had available baseline echocardiography as well as serum lyso-Gb3 results and had received ERT for more than 12 months were enrolled in this study. Informed consents were obtained from patients or from patient's parents if they were younger than 18 years. The study was approved by the medical ethics committee of Taipei Veterans General Hospital, Taiwan.

\section{Study protocol}

All patients received ERT every 2 weeks with agalsidase beta $(1 \mathrm{mg} / \mathrm{kg})$ before 2010 and agalsidase alfa $(0.2 \mathrm{mg} / \mathrm{kg})$ since 2010, due to the shortage of agalsidase beta in Taiwan [17]. A comprehensive physical examination was carried out, echocardiography and serum lyso-Gb3 were evaluated prior to and during the follow-up. Patients were divided into four groups - classical male FD, classical female FD, IVS4 + 919 G > A male FD and IVS4 + 919 G > A female FD. Longitudinal results of LVMI and serum lysoGb3 were evaluated and compared between the baseline, first and latest follow-up after ERT.

\section{Echocardiography}

Transthoracic echocardiographies were performed in accordance with American Society of Echocardiography (ACUSON Antares, Siemens, Germany; Sono 7500, Hewlett-Packard, USA). Standard two-dimensional MMode echocardiography was used from the left ventricular short axis view just below the mitral valve tip for diastolic interventricular septal thickness (IVSd), systolic and diastolic left ventricular internal diameter (LVIDs and LVIDd), and diastolic left ventricular posterior wall thickness (LVPWd). LVM was calculated according to the American Society of Echocardiography's suggestion with the following equation: $\operatorname{LVM}(\mathrm{g})=0.8 \times(1.04 \times$ $\left.[(\text { LVIDd })+(\text { IVSd })+(\text { LVPWd })]^{3}-[\text { LVIDs }]^{3}\right)+0.6[18]$. LVM was normalized to height (meter) to 2.7 power (LVMI = LVM/ height ${ }^{2.7}$ ). Normal LVM was defined as $35 \pm$ $8 \mathrm{~g} / \mathrm{m}^{2.7}$ in men and $32 \pm 8 \mathrm{~g} / \mathrm{m}^{2.7}$ in women. $\mathrm{LVH}$ was defined as LVMI $>51 \mathrm{~g} / \mathrm{m}^{2.7}$ in men and LVMI $>48 \mathrm{~g} / \mathrm{m}^{2.7}$ in women [19].

\section{Serum lyso-Gb3}

The preparation and analysis of the samples was described in our previous publication [13]. Control samples were obtained from 31 healthy adults (16 males and 15 females). Serum lyso-Gb3 level $<0.5 \mathrm{nM}$ was defined normal. All the individuals in the control group had normal enzyme activity and no known GLA gene mutation.
Patient's blood was used for lyso-Gb3 analysis. Plasma samples with $50 \mu \mathrm{L}$ or calibration standards and internal standards were added into 96 wells plate. Lipid was extracted from plasma via chloroform, methanol and formic acid. Centrifuging was carried out several times during the extraction. Stepwise gradient elusion was done via a liquid chromatography-mass spectrometry system (Waters Alliance 2795XE HPLC, USA). Tandem mass spectrometry detection of lyso-Gb3 was performed on a triple quadruple mass spectrometer (Quattro Ultima, Waters, Milford, MA) with NeoLynx software version 4.1. Multiple reaction monitoring mode (MRM) was used for lyso-GB3 measurement.

In lyso-Gb3 analysis, we used lower (5 nM) and higher (100 nM) lyso-Gb3 spiked plasma as quality controls. Our laboratory's coefficient of variation $(\mathrm{CV})$ of inter-assay was 9.2\% for the upper control and $11.0 \%$ for the lower control. As for intra-assay's CV, it was $12.8 \%$ for the upper control and $9.9 \%$ for the lower control.

\section{Anti-agalsidase antibody}

Blood was sent to SHIRE for anti-agalsidase antibodies examination. Specimens were screened and confirmed by a plate assay based upon ELISA technology, in which each patient's sample was compared to the patient's baseline. The ELISA which detected antibodies to Replagal is capable of detecting immunoglobulin isotype G (IgG) and E (IgE). Antibody positive cutpoint was absorbance ratio (time point/baseline) $\geqq 2.0$ and time point absorbance $\geqq$ 0.040. In the absence of patient baseline, normal serum was used. The neutralizing antibody (Nab) positive cutpoint was $>18 \%$ inhibition for Replagal. The lowest titer was 20 .

\section{Statistical analysis}

All statistical analyses were performed by Sigmastat version 3.5 (Dunas software LTD, Germany). The patients were divided into male and female groups and further divided into classical and IVS4 + 919G > A mutation groups. The continuous variables were compared using the paired student $t$ test or Wilcoxon Signed Rank Test between the baseline, first and last follow-up data for LVMI and serum lyso-Gb3 level. Correlation analysis had been done via linear regression between the change of serum lyso-Gb3 and the change of LVMI from the baseline and last follow-up in all the included FD patients, classical FD patients, IVS4 + 919G > A FD patients, all males and all females regardless of the mutations. $\mathrm{P}<0.05$ was considered as statistically significant.

\section{Results}

\section{Study population}

Thirty-six patients (18 males and 18 females) were included in our study. Thirteen patients were classical FD (2 males and 11 females; c.1034 C > G: 1, c.394 G > A: 2, 
Table 1 The LVMI and serum lyso-Gb3 changes after ERT in classical Fabry disease male and female patients

\begin{tabular}{lllll}
\hline Classical FD patients & Parameters, patient numbers & Baseline (Mean \pm SD) & After ERT (Mean \pm SD) & P value \\
\hline Male & LVMI (g/H2.7) N =2 & $57.2 \pm 0.1$ & $46.8 \pm 1.0$ & $0.037^{*}$ \\
& Compare between baseline and 1st Lyso-Gb3 (nM) N=2 & $191.0 \pm 17.9$ & $76.1 \pm 20.1$ & $0.009^{*}$ \\
& Compare between baseline and the last Lyso-Gb3 (nM) N=2 & $191.0 \pm 17.9$ & $80.3 \pm 15.3$ & $0.010^{*}$ \\
Female & LVMI (g/H2.7) N =11 & $65.8 \pm 30.1$ & $62.6 \pm 36.9$ & 0.533 \\
& LVMI (g/H2.7) N =6 6* & $66.7 \pm 15.6$ & $53.7 \pm 17.3$ & $0.016^{*}$ \\
& Compare between baseline and 1st Lyso-Gb3 (nM) N=11 & $16.3 \pm 8.9$ & $11.7 \pm 6.2$ & $0.005^{*}$ \\
& Compare between baseline and the last Lyso-Gb3 (nM) N=11 & $16.3 \pm 8.9$ & $11.7 \pm 7.0$ \\
\hline
\end{tabular}

*Asterisk represents statistically significant difference between the data of baseline and that after ERT ( $p<0.05)$. ERT: enzyme replacement therapy; FD: Fabry disease; LVH: left ventricular hypertrophy; LVMI: left ventricular mass index; lyso-Gb3: globotriaosylsphingosine; SD: standard deviation.

${ }^{* *}$ Case 3,4,6 were excluded in classical female FD's LVMI comparison because these patient's LVMIs were within normal limit at baseline. Case 12 and case 13 were excluded due to having risk factors for LVH.

c.1081 G> T: 1, c.612 G > A: 6, c.1194delA: 3). Twentythree patients had the IVS4 + 919G > A mutation (16 males and 7 females). The mean age was 57.8 years (range: 18.2-81.2 years). The follow-up time was 13-46 months (median: 20 months). Four of the FD patients (case 1, 14, 15 , and 24) had received agalsidase beta before June 2009 and then changed to agalsidase alfa due to the shortage of agalsidase beta supply worldwide [17]. All of other patients only received agalsidase alfa treatment.

Eighteen patients (50\%) had a history of hypertension; all of them received anti-hypertensive treatment and were normotensive $(<140 / 90 \mathrm{mmHg})$ before receiving ERT. Six

\section{(A) Classical FD, male, LVMI change}

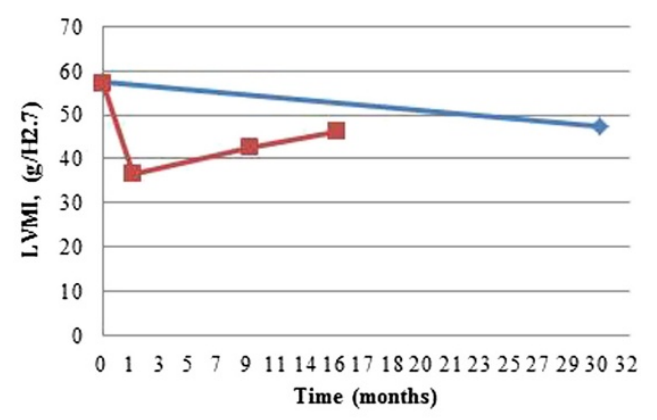

\section{(C) Classical FD, female, LVMI change}

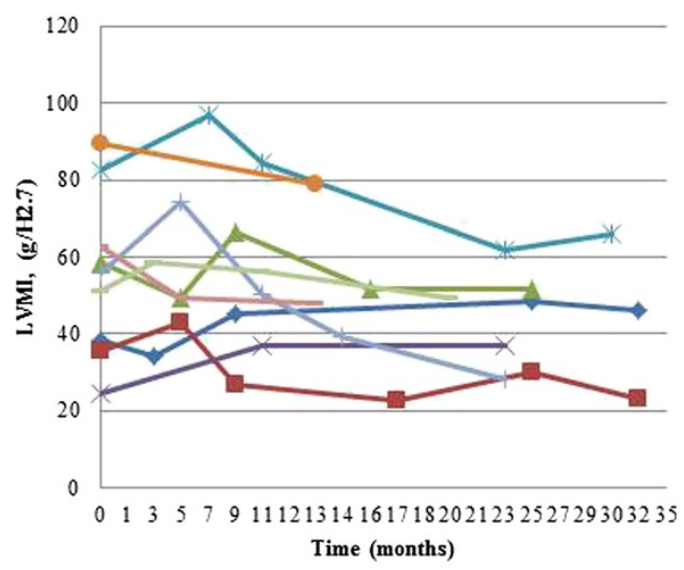

\section{Classical FD, male, Lyso-GB3 change}

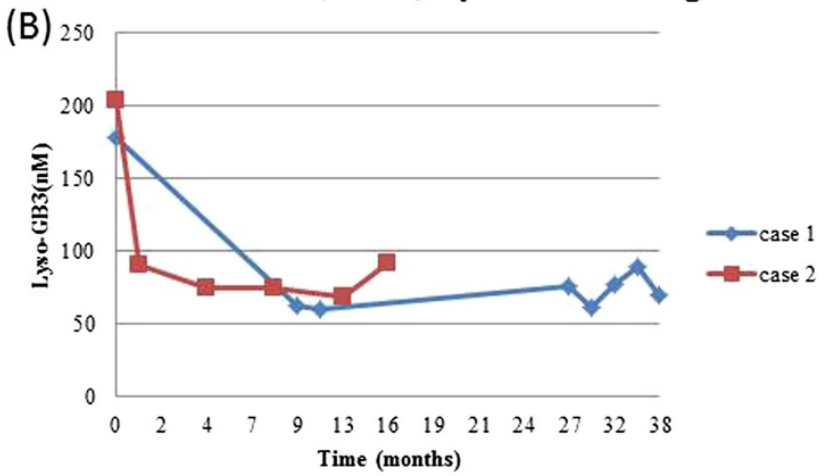

(D) Classical FD, female, Lyso-GB3 change

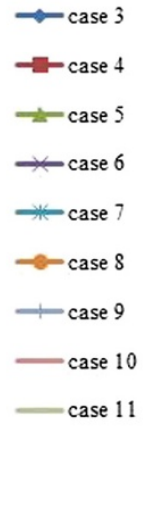

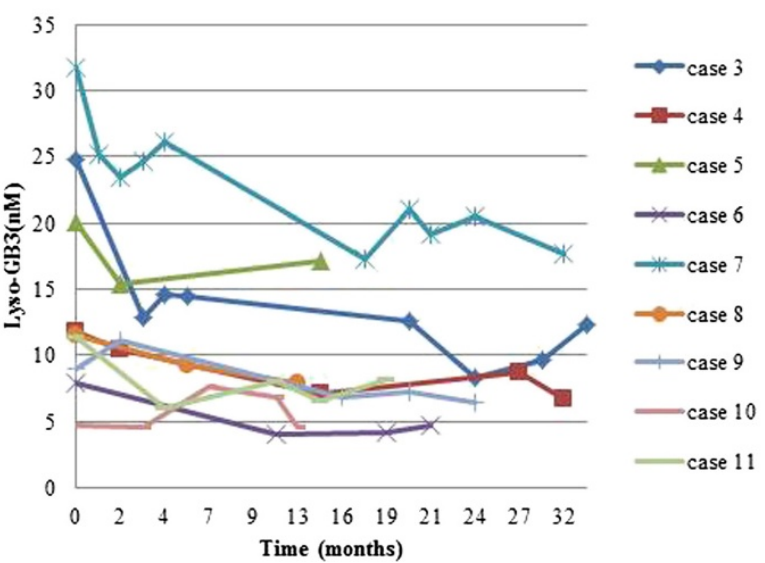

Figure 2 The LVMI and serum lyso-Gb3 change after ERT in classical Fabry disease male (A) (B) and female (C) (D) patients. Case 12 and case 13 were excluded due to having risk factors for left ventricular hypertrophy. FD: Fabry disease; LVMI: left ventricular mass index; lyso-Gb3: globotriaosylsphingosine. 


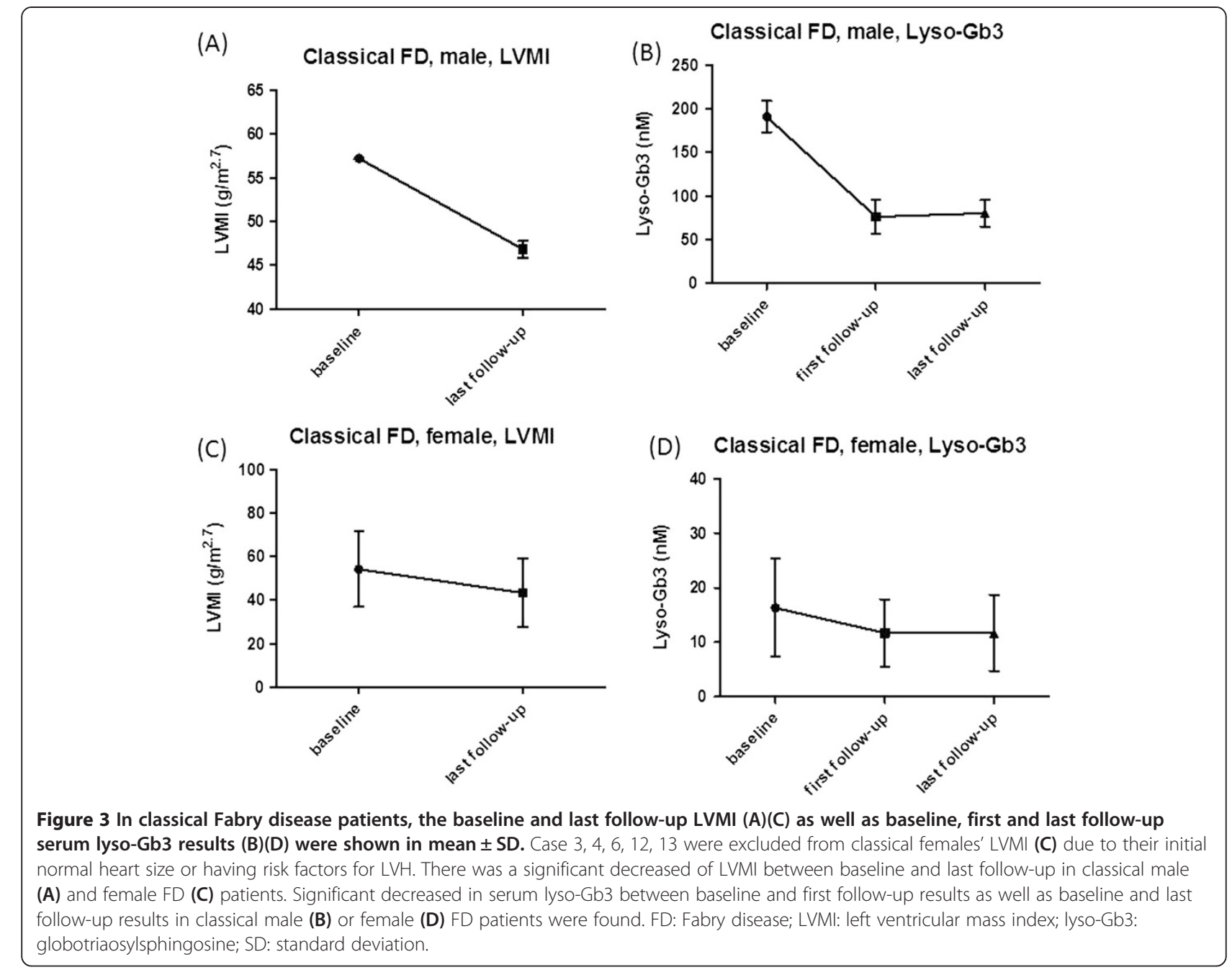

patients (16.7\%) had a history of diabetes mellitus; five of them received oral hypoglycemic agents, one of them had regular insulin injections. Four patients (11.1\%) had atrial fibrillation under medical control. Three patients (8.3\%) had sick sinus syndrome and had received pacemaker implantation. One patient had lung adenocarcinoma and had received a complete course of chemotherapy. One patient stopped ERT by herself due to the improvement of $\mathrm{LVH}$ but re-started ERT owing to a remarkably elevated lysoGb3 level after ERT was discontinued. One patient had congestive heart failure which was under medical control.

\section{LVMI}

The overall LVMI was reduced significantly (LVMI: $70.9 \pm$ $28.7 \mathrm{~g} / \mathrm{m}^{2.7}$ vs. $61.5 \pm 28.6 \mathrm{~g} / \mathrm{m}^{2.7}, \mathrm{p}<0.001$ ) after ERT while on follow-up. On sub-group analysis, LVMI was reduced significantly in both genders (male: $78.5 \pm$ $31.2 \mathrm{~g} / \mathrm{m}^{2.7}$ vs. $68.3 \pm 25.3 \mathrm{~g} / \mathrm{m}^{2.7}, \mathrm{p}=0.007$; female: $63.3 \pm 24.5 \mathrm{~g} / \mathrm{m}^{2.7}$ vs. $\left.54.6 \pm 30.7 \mathrm{~g} / \mathrm{m}^{2.7}, \mathrm{p}=0.023\right)$. In the classical male group, both of the patients had a significantly reduced LVMI $\left(57.2 \pm 0.1 \mathrm{~g} / \mathrm{m}^{2.7}\right.$ vs. $46.8 \pm$ $\left.1.0 \mathrm{~g} / \mathrm{m}^{2.7}, \mathrm{p}=0.037\right)$. In the classical female group, interestingly, the initial analysis showed that the LVMI did not improve significantly (LVMI: $65.8 \pm 30.1 \mathrm{~g} / \mathrm{m}^{2.7}$ vs. $\left.62.6 \pm 36.9 \mathrm{~g} / \mathrm{m}^{2.7}, \mathrm{p}=0.533\right)$. However, three out of 9 female classical patients did not have LVH at the beginning of ERT (case 3, 4, and 6). One other classical female (case 13) had congestive heart failure before ERT and another classical female (case 12) had a non-ST segment elevation myocardial infarction (NSTEMI) during the course of ERT. The episode of NSTEMI was proved by the typical history of chest pain radiated to the back, $\mathrm{T}$ wave inversion over the inferior leads in electrocardiogram, and elevated serum troponin I which decreased gradually after management. Both of the conditions are risk factors for LVH. After excluding these 5 female patients, there was a significant improvement of LVMI in the classical female group. (LVMI: $66.7 \pm$ $15.6 \mathrm{~g} / \mathrm{m}^{2.7}$ vs. $55.47 \mathrm{~g} / \mathrm{m}^{2.7}$, range: $53.7 \pm 17.3 \mathrm{~g} / \mathrm{m}^{2.7}, \mathrm{p}=$ 0.016) (Table 1). 


\section{Serum lyso-Gb3 analysis}

After receiving ERT, the next serum lyso-Gb3 level was significantly decreased when compared with the baseline level in all groups (male FD: $8.91 \mathrm{nM}$, range: 2.81-203.68 $\mathrm{nM}$ vs. $6.74 \mathrm{nM}$, range: $3.79-90.31 \mathrm{nM}, \mathrm{p}=0.002$; female FD: $11.0 \pm 9.7 \mathrm{nM}$ vs. $8.0 \pm 7.6 \mathrm{nM}, \mathrm{p}=0.004$; classical male FD: $191.0 \pm 17.9 \mathrm{nM}$ vs. $76.1 \pm 20.1, \mathrm{p}=0.009$; classical female FD: $16.3 \pm 8.9 \mathrm{nM}$ vs. $11.7 \pm 6.2 \mathrm{nM}, \mathrm{p}=$ 0.005 ; IVS4 + 919 G > A male FD: $8.5 \pm 3.3$ nM vs. $6.7 \pm$ $2.2 \mathrm{nM}, \mathrm{p}=0.012$; IVS $4+919 \mathrm{G}>$ A female FD: $2.6 \pm 1.0$ $\mathrm{nM}$ vs. $1.2 \pm 0.9 \mathrm{nM}, \mathrm{p}=0.018$ ). (Figures 2, 3, 4 and 5)

However, in both males and females with the IVS4+ 919G > A mutation, the lyso-Gb3 level was found to be increasing slightly even when the LVMI of these patients was still improving or remaining stable after a longer follow-up time.(Figures 4 and 5) Therefore, we compared the baseline and the last serum lyso-Gb3 level in both male and female groups and found the significant differences in lyso-Gb3 levels disappeared between the baseline and the last follow-up (IVS4 + 919G > A male FD: $8.5 \pm 3.3 \mathrm{nM}$ vs. $7.4 \pm 2.8 \mathrm{nM}, \mathrm{p}=0.163$; IVS4 +
919G > A female FD: $2.6 \pm 1.0 \mathrm{nM}$ vs. $2.6 \pm 1.6 \mathrm{nM}, \mathrm{p}=$ $0.938)$ even when the significant differences in LVMIs still existed between the baseline and the last follow-up (IVS4 + 919G > A male FD: $81.1 \pm 32.2 \mathrm{~g} / \mathrm{m}^{2.7}$ vs. $71.0 \pm$ $25.6 \mathrm{~g} / \mathrm{m}^{2.7}, \mathrm{p}=0.016$; IVS4 + 919G > A female FD: $59.4 \pm$ $12.8 \mathrm{~g} / \mathrm{m}^{2.7}$ vs. $42.0 \pm 10.2 \mathrm{~g} / \mathrm{m}^{2.7}, \mathrm{p}<0.001$ ) (Table 2). We found that there was a trend in lyso-Gb3 levels during long-term ERT for most of our patients. In the beginning, there was a good response of lyso-Gb3 to ERT. The mean lowest serum lyso-Gb3 level was found around 11.1 months (median: 9 months) after ERT and then lyso-Gb3 level elevated gradually, even when the LVMI was still reducing or remaining stable. Therefore, at the last follow-up, compared with baseline, the significant differences in lyso-Gb3 levels had disappeared, but the significant differences in LVMI still existed.

Similarly, we also found the classical patients had a very good response in lyso-Gb3 levels during the early period of ERT. The mean lowest serum lyso-Gb3 level was found around 13.3 months after ERT. Thereafter,

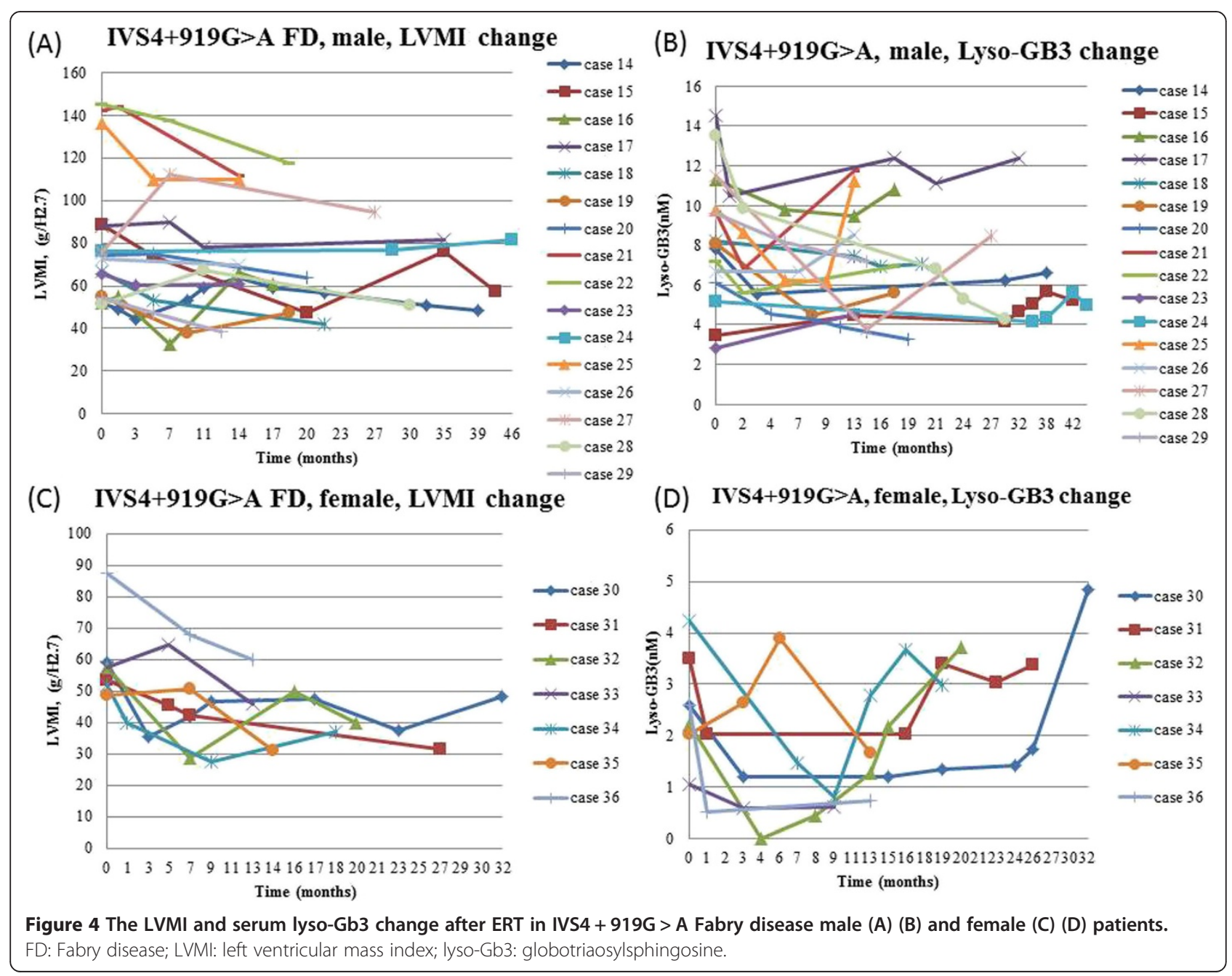




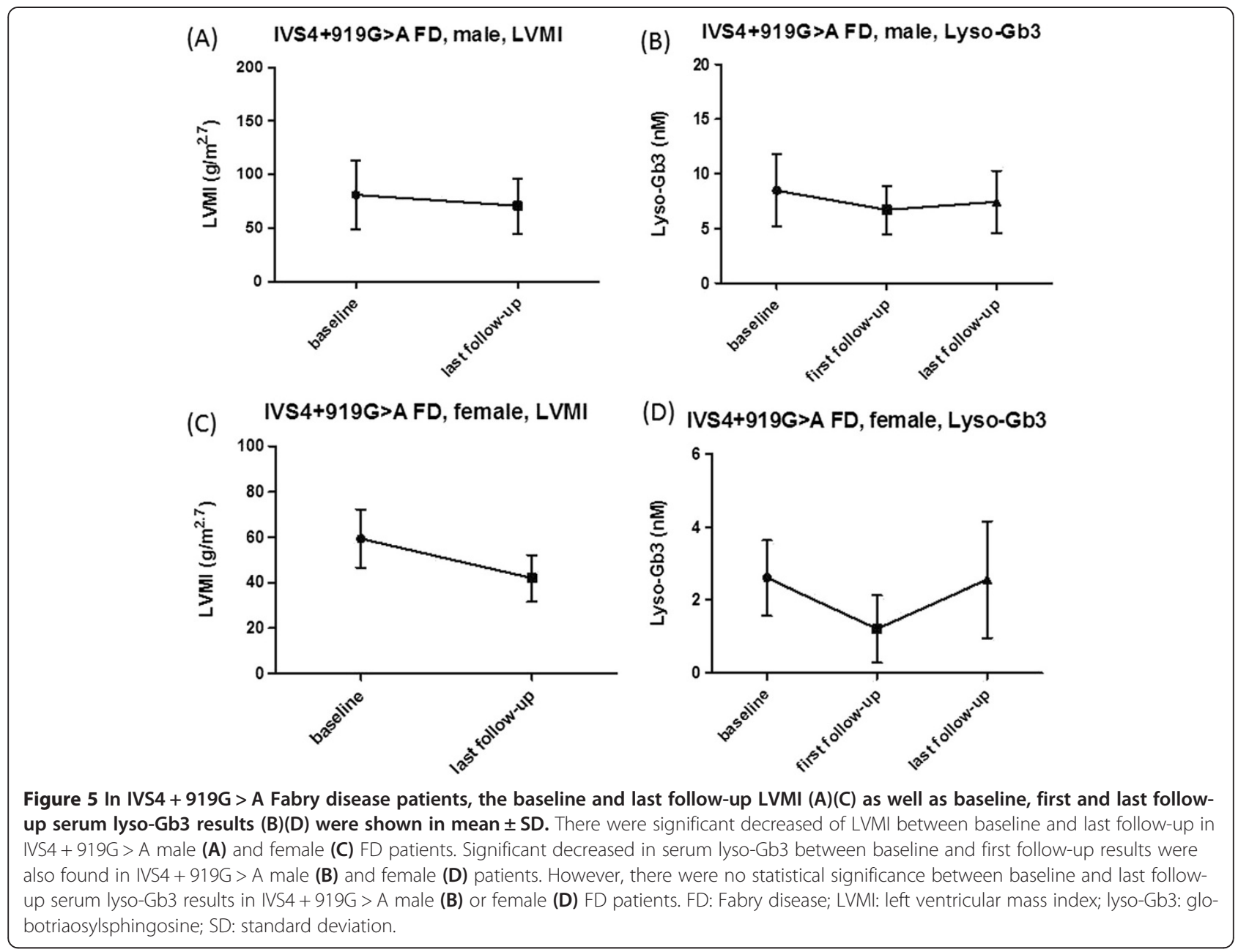

the lyso-Gb3 level started to increase gradually, but the increase was not as significant as in our cardiac variant patients.

\section{Anti-agalsidase alfa antibody analysis}

Twenty-three patients received serum anti-agalsidase alfa analysis. Only one classical male (case 1 ) and one classical female (case 12) had positive anti-agalsidase alfa IgG, but IgE and NAb were all negative in both of the patients. None of the IVS4+919G > A patients had anti-agalsidase alfa antibody.

\section{Correlation analysis between serum lyso-Gb3 and LVMI}

There were no significant correlation between the change of LVMI and the change of serum lyso-Gb3 from baseline to the last follow-up in all the included FD patients $(\mathrm{r}=0.002, \mathrm{p}=0.912)$, classical FD patients $(\mathrm{r}=0.191, \mathrm{p}=0.531)$, IVS4 $+919 \mathrm{G}>\mathrm{A}$ FD patients $(\mathrm{r}=$ $0.364, \mathrm{p}=0.088)$, all males $(\mathrm{r}=0.024, \mathrm{p}=0.926)$ and all females $(r=0.167, p=0.51)$ regardless of the mutations.

\section{Discussion}

From our Fabry newborn screening program, we found a surprisingly high prevalence of a GLA mutation, IVS4 + 919G $>A$, in our population (1/875 in males and 1/399 in females) [11]. In addition to Taiwan, this mutation was also found in Mainland China, Singapore (Han population), and Malaysia (Han population) (unpublished data). Therefore, we believe the IVS4 + 919G > A mutation may be the most common pathogenic mutation of FD in the world. It is very important to understand the natural course of the disease caused by this mutation, as well as treatment outcomes and suitable biomarkers for monitoring ERT.

Lyso-Gb3, a deacylated Gb3, has been shown to have a proliferative effect on smooth muscle cells. Elevated lysoGB3 levels have been found in classical and late-onset FD patients [16,20-23]. ERT can dramatically decrease serum lyso-Gb3 levels. Rombach et al. found a relationship between serum lyso-Gb3 level and the severity of classical FD [20,21]. In our previous study, we found LVMI and lyso-Gb3 levels in patients with IVS4 + 919G > A mutation had a good response to ERT during the early treatment 
Table 2 The LVMI and serum lyso-Gb3 change after ERT in IVS4 + 919G > A Fabry disease male and female patients

\begin{tabular}{|c|c|c|c|c|}
\hline Cardiac variant, IVS4 + 919G > A FD patients & Parameters, patient number & Baseline (Mean \pm SD) & After ERT (Mean \pm SD) & $P$ value \\
\hline \multirow[t]{3}{*}{ Male } & LVMI $\left(\mathrm{g} / \mathrm{H}^{2.7}\right) \mathrm{N}=16$ & $81.1 \pm 32.2$ & $71.0 \pm 25.6$ & $0.016^{*}$ \\
\hline & $\begin{array}{l}\text { Compare between baseline } \\
\text { and } 1^{\text {st }} \text { Lyso-Gb3 }(\mathrm{nM}) \mathrm{N}=16\end{array}$ & $8.5 \pm 3.3$ & $6.7 \pm 2.2$ & $0.012^{*}$ \\
\hline & $\begin{array}{l}\text { Compare between baseline and } \\
\text { the last Lyso-Gb3 (nM) N =16 }\end{array}$ & $8.5 \pm 3.3$ & $7.4 \pm 2.8$ & 0.163 \\
\hline \multirow[t]{3}{*}{ Female } & LVMI $\left(g / H^{2.7}\right) N=7$ & $59.4 \pm 12.8$ & $42.0 \pm 10.2$ & $<0.001^{*}$ \\
\hline & $\begin{array}{l}\text { Compare between baseline } \\
\text { and } 1^{\text {st }} \text { Lyso-Gb3 (nM) N=7 }\end{array}$ & $2.6 \pm 1.0$ & $1.2 \pm 0.9$ & $0.018^{*}$ \\
\hline & $\begin{array}{l}\text { Compare between baseline and } \\
\text { the last Lyso-Gb3 (nM) N=7 }\end{array}$ & $2.6 \pm 1.0$ & $2.6 \pm 1.6$ & 0.938 \\
\hline
\end{tabular}

${ }^{*}$ Asterisk represents statistically significant difference between the data of baseline and that after ERT ( $\left.p<0.05\right)$. ERT: enzyme replacement therapy; FD: Fabry disease; LVMI: left ventricular mass index; lyso-Gb3: globotriaosylsphingosine; SD: standard deviation.

period [16]. However, the responses to a longer-term treatment have not been previously analyzed. In this study, we analyzed the long-term responses of LVMI and lyso-Gb3 to ERT in our patients with IVS4 +919G > A mutation. To our surprise, the significant difference in lyso-Gb3 levels disappeared gradually between baseline and followup after long-term ERT, even when the LVMI of these patients were still improving or remaining stable. The reasons for the elevation of lyso-Gb3 levels remain unknown. We suspect that there are some tissues, which might not cause significant manifestations of FD, that respond poorly to ERT and the accumulation of lyso-Gb3 was still in progress. Furthermore, lyso-Gb3 is only very marginally elevated in our IVS4 + 919G > A patients. Due to the very low range, there might be an unacceptable degree of assay variation, which would render this marker unsuitable for follow-up.

For classical patients, we did not find as significant an elevation of lyso-Gb3 levels as in patients with IVS4 + 919G > A mutation during long-term follow-up. Because the lyso-Gb3 levels of classical patients were much higher than those with IVS4 + 919G > A mutations, we suspect that the amount of lyso-Gb3 accumulation in the tissues responding poorly to ERT was too small to affect the serum lyso-Gb3 levels.

When we further analyzed the correlation between the change of LVMI and the change of serum lyso-Gb3 in different FD patient groups, we found no significant correlations between the changes of these two parameters while longer follow-up. It means a mark decreased serum lysoGb3 does not indicate a greater improvement of cardiac size. Although Rombach et al. [21] had reported a good correlation between plasma lyso-Gb3 and LVM in female classical FD patients before ERT, serum lyso-Gb3 cannot be used as a long-term monitor for the change of LVMI in both classical and cardiac variant FD patients after ERT.

Our study reveals that serum lyso-Gb3 could be a good biomarker for the response to ERT in the early stages of treatment for patients with the IVS4 + 919G >
A mutation. However, serum lyso-Gb3 loses its reliability for monitoring the therapeutic outcome to ERT in these cardiac variant patients.

There were several limitations in this study. First, the number of patients in each group was not large enough, especially for the classical patients, to make a solid conclusion. Although the echocardiographic examination was performed via a standard protocol, employing a single echocardiographic machine and only two skilled cardiologists, the variability of LVMI measurements still needs to be considered. Furthermore, the serum lyso-Gb3 levels of the patients with IVS4 + 919G > A mutation were very low. Although we did our best to maintain experimental stability and consistency and kept our intra-assay and inter-assay $\mathrm{CV}$ values within the acceptable range, the $\mathrm{CV}$ of our laboratory still had an influence on the interpretation of the results.

\section{Conclusion}

Even lyso-Gb3 has a high diagnostic sensitivity in FD patients and has a good response to ERT in early stages, it might not be a reliable follow-up marker for monitoring the long-term therapeutic result of ERT in late-onset FD patients with the Chinese hotspot mutation, IVS4 + 919G > A.

\section{Abbreviations}

a-Gal A: a-galactosidase A; CV: Coefficient of variation; ERT: Enzyme replacement therapy; FD: Fabry disease; Gb3: Globotriaosylceramide; IgG: Immunoglobulin isotype G; IgE: Immunoglobulin isotype E; IVSd: Diastolic interventricular septal thickness; LVH: Left ventricular hypertrophy; LVIDd: Diastolic left ventricular internal diameter; LVIDs: Systolic left ventricular internal diameter; LVM: Left ventricular mass; LVMI: Left ventricular mass index; LVPWd: Diastolic left ventricular posterior wall thickness; Lyso-Gb3: Globotriaosylsphingosine; MRM: Multiple reaction monitoring mode; Nab: Neutralizing antibody; NSTEMI: Non-ST segment elevation myocardial infarction.

\section{Competing interests}

The authors declare that they have no competing interests. 


\section{Authors' contributions}

HCL and HYL performed acquisition, statistical analysis and interpretation of data, and drafting of the manuscript. WCY and DMN participated in design of the study, interpretation of the data and helped to draft the manuscript. FPC performed the pathological interpretation of cadiomyocytes. $\mathrm{HCL}, \mathrm{CKH}$, and YHL performed biochemical analyses and revised the manuscript. SPL, CFY, TRH, and CWL were responsible for patient screening. All authors read and accepted the manuscript.

\section{Acknowledgements}

The authors thank SHIRE human Genetics Therapies for Anti-agalsidase alfa antibody analysis. This study was partially supported by the National Science Council, Taiwan (NSC-102-2314-B-195-017-MY3) and Taipei Veterans General Hospital (V101C-129). Our center has received several research grants regarding Fabry disease from Shire Pharmaceuticals and Excelsior Biopharma. SHIRE provided English editing of this manuscript, however, SHIRE had no role in the study design, data collection, analysis, and interpretation. The opinions of this study remain with the authors.

\section{Author details}

'Department of Pediatrics, Taipei Veterans General Hospital, No. 201, Section 2, Shih-Pai Road, Taipei 112, Taiwan. ${ }^{2}$ Taiwan Clinical Trial Consortium in Fabry Disease, Taipei, Taiwan. ${ }^{3}$ Institute of Clinical Medicine, National Yang-Ming University, Taipei, Taiwan. ${ }^{4}$ Department of Pediatrics, Mackay Memorial Hospital, Taipei, Taiwan. ${ }^{5}$ Department of Medicine, Mackay Medical College, New Taipei City, Taiwan. ${ }^{6}$ Mackay Junior College of Medicine, Nursing and Management, Taipei, Taiwan. ${ }^{7}$ Neonatal Screening Center, Chinese Foundation of Health, Taipei, Taiwan. ${ }^{8}$ Department of Pediatrics, Cathay General Hospital, Taipei, Taiwan. ${ }^{9}$ Pathology and Laboratory Medicine Department, Taipei Veterans General Hospital, Taipei, Taiwan. ${ }^{10}$ Division of Cardiology, Department of Medicine, Taipei Veterans General Hospital, No. 201, Section 2, Shih-Pai Road, Taipei 112, Taiwan. ${ }^{11}$ National Yang-Ming University, School of Medicine, No. 201, Section 2, Shih-Pai Road, Taipei 112, Taiwan.

Received: 28 March 2014 Accepted: 3 July 2014

Published: 22 July 2014

\section{References}

1. Desnick RJ, loannou YA, Eng CM: a-Galactosidase A deficiency: Fabry disease. In The Metabolic and Molecular Bases of Inherited Disease. Volume 3. 8th edition. Edited by Scriver CR, Sly WS, Childs B, Beaudet AL, Valle D, Kinzler KW, Vogelstein B. New York: McGraw-Hill; 2001:3733-3774.

2. Zarate YA, Hopkin RJ: Fabry's disease. Lancet 2008, 372:1427-1435.

3. Meikle PJ, Hopwood JJ, Clague AE, Carey WF: Prevalence of lysosomal storage disorders. JAMA 1999, 281:249-254.

4. Nakao S, Takenaka T, Maeda M, Kodama C, Tanaka A, Tahara M, Yoshida A, Kuriyama M, Hayashibe H, Sakuraba H, Tanaka H: An atypical variant of Fabry's disease in men with left ventricular hypertrophy. N Engl J Med 1995, 333:288-293.

5. Kotanko P, Kramar R, Devrnja D, Paschke E, Voigtlander T, Auinger M, Pagliardini S, Spada M, Demmelbauer K, Lorenz M, Hauser AC, Kofler HJ, Lhotta K, Neyer U, Pronai W, Wallner M, Wieser C, Wiesholzer M, Zodl H, Födinger M, Sunder-Plassmann G: Results of a nationwide screening for Anderson-Fabry disease among dialysis patients. J Am Soc Nephrol 2004, 15:1323-1329

6. Rolfs A, Bottcher T, Zschiesche M, Morris P, Winchester B, Bauer P, Walter U, Mix E, Lohr M, Harzer K, Strauss U, Pahnke J, Grossmann A, Benecke R: Prevalence of Fabry disease in patients with cryptogenic stroke: a prospective study. Lancet 2005, 366:1794-1796.

7. Monserrat L, Gimeno-Blanes JR, Marin F, Hermida-Prieto M, Garcia-Honrubia A, Perez I, Fernandez X, de Nicolas R, de la Morena G, Paya E, Yague J, Egido J: Prevalence of fabry disease in a cohort of 508 unrelated patients with hypertrophic cardiomyopathy. J Am Coll Cardiol 2007, 50:2399-2403.

8. Sachdev B, Takenaka T, Teraguchi H, Tei C, Lee P, McKenna WJ, Elliott PM: Prevalence of Anderson-Fabry disease in male patients with late onset hypertrophic cardiomyopathy. Circulation 2002, 105:1407-1411.

9. Chong KW, Lu YH, Hsu JH, Lo MY, Hsiao CY, Niu DM: High incidence of cardiac variant of Fabry disease in Taiwanese revealed by newborn screening. Taiwan Hum Genet Soc Autumn Symp 2008, 1:92-98.

10. Lin HY, Chong KW, Hsu JH, Yu HC, Shih CC, Huang CH, Lin SJ, Chen CH, Chiang CC, Ho HJ, Lee PC, Kao CH, Cheng KH, Hsueh C, Niu DM: High incidence of the cardiac variant of Fabry disease revealed by newborn screening in the Taiwan Chinese population. Circ Cardiovasc Genet 2009, 2:450-456.

11. Chien YH, Lee NC, Chiang SC, Desnick RJ, Hwu WL: Fabry disease: incidence of the common later-onset alpha-galactosidase A IVS4 + 919G- > A mutation in Taiwanese newborns-superiority of DNA-based to enzyme-based newborn screening for common mutations. Mol Med 2012, 18:780-784.

12. Tai CL, Liu MY, Yu HC, Chiang CC, Chiang H, Suen JH, Kao SM, Huang YH, Wu TJ, Yang CF, Tsai FC, Lin CY, Chang JG, Chen HD, Niu DM: The use of high resolution melting analysis to detect Fabry mutations in heterozygous females via dry bloodspots. Clin Chim Acta 2012, 413:422-427.

13. Liao HC, Huang YH, Chen YJ, Kao SM, Lin HY, Huang CK, Liu HC, Hsu TR, Lin SP, Yang CF, Fann CS, Chiu PC, Hsieh KS, Fu YC, Ke YY, Lin CY, Tsai FJ, Wang CH, Chao MC, Yu WC, Chiang CC, Niu DM: Plasma globotriaosylsphingosine (lysoGb3) could be a biomarker for Fabry disease with a Chinese hotspot late-onset mutation (IVS4+919G>A). Clin Chim Acta 2013, 426C:114-120.

14. Togawa T, Kawashima I, Kodama T, Tsukimura T, Suzuki T, Fukushige T, Kanekura T, Sakuraba H: Tissue and plasma globotriaosylsphingosine could be a biomarker for assessing enzyme replacement therapy for Fabry disease. Biochem Biophys Res Commun 2010, 399:716-720.

15. van Breemen MJ, Rombach SM, Dekker N, Poorthuis BJ, Linthorst GE, Zwinderman AH, Breunig F, Wanner C, Aerts JM, Hollak CE: Reduction of elevated plasma globotriaosylsphingosine in patients with classic Fabry disease following enzyme replacement therapy. Biochim Biophys Acta 1812, 2011:70-76.

16. Lin HY, Liu HC, Huang YH, Liao HC, Hsu TR, Shen Cl, Li ST, Li CF, Lee LH, Lee PC, Huang CK, Chiang CC, Lin CY, Lin SP, Niu DM: Effects of enzyme replacement therapy for cardiac-type Fabry patients with a Chinese hotspot late-onset Fabry mutation (IVS4 + 919G > A). BMJ Open 2013, 3:e003146. doi:10.1136/bmjopen-2013-003146.

17. Lin HY, Huang YH, Liao HC, Liu HC, Hsu TR, Shen Cl, Li ST, Li CF, Lee LH, Lee PC, Huang CK, Chiang CC, Lin SP, Niu DM: Clinical observations on enzyme replacement therapy in patients with Fabry disease and the switch from agalsidase beta to agalsidase alfa. J Chin Med Assoc 2014, 77:190-197.

18. Lang RM, Bierig M, Devereux RB, Flachskampf FA, Foster E, Pellikka PA, Picard MH, Roman MJ, Seward J, Shanewise JS, Solomon SD, Spencer KT, Sutton MS, FASE, Stewart WJ: Recommendations for chamber quantification: a report from the American Society of Echocardiography's Guidelines and Standards Committee and the Chamber Quantification Writing Group, developed in conjunction with the European Association of Echocardiography, a branch of the European Society of Cardiology. J Am Soc Echocardiogr 2005, 18:1440-1463.

19. de Simone G, Daniels SR, Devereux RB, Meyer RA, Roman MJ, de Divitiis O, Alderman MH: Left ventricular mass and body size in normotensive children and adults: assessment of allometric relations and impact of overweight. J Am Coll Cardiol 1992, 20:1251-1260.

20. Aerts JM, Groener JE, Kuiper S, Donker-Koopman WE, Strijland A, Ottenhoff R, van Roomen C, Mirzaian M, Wijburg FA, Linthorst GE, Vedder AC, Rombach SM, Cox-Brinkman J, Somerharju P, Boot RG, Hollak CE, Brady RO, Poorthuis BJ: Elevated globotriaosylsphingosine is a hallmark of Fabry disease. Proc Natl Acad Sci U S A 2008, 105:2812-2817.

21. Rombach SM, Dekker N, Bouwman MG, Linthorst GE, Zwinderman AH, Wijburg FA, Kuiper S, Vd Bergh Weerman MA, Groener JE, Poorthuis BJ Hollak CE, Aerts JM: Plasma globotriaosylsphingosine: diagnostic value and relation to clinical manifestations of Fabry disease. Biochim Biophys Acta 2010, 1802:741-748.

22. Togawa T, Kodama T, Suzuki T, Sugawara K, Tsukimura T, Ohashi T, Ishige N, Suzuki K, Kitagawa T, Sakuraba H: Plasma globotriaosylsphingosine as a biomarker of Fabry disease. Mol Genet Metab 2010, 100:257-261.

23. Chien YH, Bodamer OA, Chiang SC, Mascher H, Hung C, Hwu WL: Lyso-globotriaosylsphingosine (lyso-Gb(3)) levels in neonates and adults with the Fabry disease later-onset GLA IVS4 + 919G > A mutation. $J$ Inherit Metab Dis 2013, 36:881-885.

\section{doi:10.1186/s13023-014-0111-y}

Cite this article as: Liu et al:: Globotriaosylsphingosine (lyso-Gb3) might not be a reliable marker for monitoring the long-term therapeutic outcomes of enzyme replacement therapy for late-onset Fabry patients with the Chinese hotspot mutation (IVS4+919G>A). Orphanet Journal of Rare Diseases 2014 9:111. 